\title{
Associação entre Atividade Física no Tempo Livre e HDL-C em Participantes do Elsa-Brasil: Existem Diferenças entre Homens e Mulheres no Efeito Dose-Resposta?
}

\author{
Association between Leisure Time Physical Activity and HDL-C in the Elsa-Brasil Study Participants: Are There \\ Any Gender Differences in the Dose-Response Effect?
}

Francisco José Gondim Pitanga, ${ }^{1 \oplus}$ Rosane Harter Griep, ${ }^{2}{ }^{\circledR}$ Maria da Conceição Almeida, ${ }^{3} @$ Maria de Jesus Mendes da Fonseca, ${ }^{4}$ Andreia Rios de Souza, ${ }^{\circledR}$ Raiane de Carvalho Silva, ${ }^{5}$ Sheila Maria Alvim Matos ${ }^{6}$

Programa de Pós-Graduação em Ciências da Reabilitação - Universidade Federal da Bahia, ${ }^{1}$ Salvador, BA - Brasil

Laboratório de Educação em Meio Ambiente e Saúde, Instituto Oswaldo Cruz, Fiocruz, ${ }^{2}$ Rio de Janeiro, RJ - Brasil

Instituto Gonçalo Moniz, ${ }^{3}$ Salvador, BA - Brasil

Escola Nacional de Saúde Pública (Fiocruz), ${ }^{4}$ Rio de Janeiro, RJ - Brasil

Universidade Federal da Bahia, ${ }^{5}$ Salvador, BA - Brasil

Instituto de Saúde Coletiva - Universidade Federal da Bahia, ${ }^{6}$ Salvador, BA - Brasil

\section{Resumo}

Fundamento: Níveis elevados de lipoproteína de alta densidade (HDL-C) podem ter efeitos positivos para proteção de agravos cardiovasculares, e prática regular de atividade física no tempo livre (AFTL) tem sido associada ao seu aumento.

Objetivo: Verificar a existência de possíveis diferenças entre homens e mulheres no efeito dose-resposta na associação entre AFTL e HDL-C.

Métodos: Estudo transversal com dados de 13,931 participantes de ambos os sexos (7,607 mulheres) do Estudo Longitudinal da Saúde do Adulto (ELSA-Brasil). A AFTL foi mensurada por meio do International Physical Activity Questionnary (IPAQ) e classificada em quatro categorias: inativos, pouco ativos, ativos e muito ativos. O poder discriminatório da AFTL para HDL-C elevado, nas diferentes intensidades analisadas (caminhada, atividade física moderada e atividade física vigorosa) foi testado por meio das curvas ROC. As associações, ajustadas por variáveis de confundimento entre AFTL e HDL-C foram analisadas por meio de regressão logística, estimando-se a odds ratio (OR) com intervalo de confiança (IC) de $\mathbf{9 5 \%}$.

Resultados: Observou-se associação positiva, com efeito dose-resposta, entre AFTL e HDL-C tanto em homens quanto em mulheres. Com relação à intensidade, apenas a atividade física vigorosa discriminou o HDL-C elevado em homens, enquanto tanto a atividade física de caminhada quanto a moderada e a vigorosa discriminaram o HDL-C elevado em mulheres.

Conclusão: A AFTL apresenta associação positiva com gradiente dose-resposta com HDL-C; no entanto, entre os homens, a associação não é observada para aqueles classificados como pouco ativos fisicamente. Nas mulheres, tanto a intensidade da caminhada quanto a atividade física moderada ou vigorosa podem discriminar níveis de HDL-C mais altos; já nos homens, essa relação é observada apenas na intensidade vigorosa.

Palavras-chave: Doenças Cardiovasculares; Exercício; Atividade Física; Estudos Transversais; HDL-Colesterol; Epidemiologia; Caminhada.

\footnotetext{
Abstract practice of leisure time physical activity (LTPA) may be associated with their increase. in the ELSA-Brasil study cohort.

Correspondência: Francisco José Gondim Pitanga •

Universidade Federal da Bahia - Av. Reitor Miguel Calmom, s/n. CEP 40110-060, Salvador, BA - Brasil

E-mail: pitanga@lognet.com.br

Artigo recebido em 07/05/2020, revisado em 13/08/2020, aceito em 09/09/2020
}

Background: High levels of high-density lipoprotein (HDL-C) are known for their protective effect against cardiovascular diseases and the regular

Objective: To verify the existence of differences between genders in the dose-response effect regarding the association between LTPA and HDL-C

Methods: Cross-sectional study with data from wave 2 of 13,931 participants of both genders (7,607 women) from the Longitudinal Study of Adult Health ELSA-Brasil. The LTPA was measured using the International Physical Activity Questionnaire (IPAQ) and classified into four categories: sedentary, low active, active and very active. The discriminatory power of LTPA at different intensities analyzed for high HDL-C was tested using ROC curves. Associations, adjusted for confounders between LTPA and HDL-C were analyzed by logistic regression. A 95\% confidence interval was used.

DOI: https://doi.org/10.36660/abc.20200438 
Results: A positive association, with a dose-response effect, was observed between LTPA and HDL-C in both men and women. With regard to intensity, only vigorous physical activity discriminated high HDL-C in men, while both walking and moderate and vigorous physical activity discriminated high HDL-C in women.

Conclusions: LTPA shows a positive association with gradient dose-response and HDL-C, but in men, the association is not observed for those classified as physically unfit. In women, both walking intensity and moderate or vigorous physical activity can discriminate high HDL-C levels, whereas only vigorous intensity-exercise discriminate elevated HDL-C levels in men, demonstrating that males need to do more physical activity for this benefit to be observed.

Keywords: Cardiovascular Diseases; Exercise; Physical Activity; Cross-Sectional Studies; HDL-Cholesterol; Epidemiology; Walking.

Full texts in English - http://www.arquivosonline.com.br

\section{Introdução}

A prática regular da atividade física (AF) apresenta associação positiva com o aumento dos níveis de lipoproteína de alta densidade (HDL-C) e redução dos níveis de triglicérides, com alterações pouco consistentes em relação aos níveis de lipoproteína de baixa densidade (LDL-C). ${ }^{1}$ Ademais, maiores níveis de HDL-C podem apresentar benefícios na proteção contra doença arterial coronariana; entretanto, estudos mais recentes de randomização mendeliana têm sugerido o HDL-C mais como um marcador de risco cardiovascular do que propriamente um fator de risco. ${ }^{2,3}$

O aumento do HDL-C em função da prática de AF pode ser explicado na medida em que, durante a mesma, há aumento da atividade da lipoproteína lipase (LPL), acelerando, assim, a quebra das lipoproteínas ricas em triglicérides. Com isso, gerase a transferência de colesterol, fosfolipídio e proteínas para as partículas nascentes do HDL-C. ${ }^{4}$ Além disso, em indivíduos mais ativos fisicamente, o tempo médio de vida do HDL-C pode alcançar 6 a 7 dias, enquanto, nos inativos fisicamente, é de apenas 3 a 4 dias. $^{5}$

Apesar dessas evidências, a intensidade ideal de AF para proporcionar o aumento do HDL-C precisa ser melhor esclarecida, principalmente com ênfase em possíveis diferenças entre homens e mulheres demonstrada em estudos prévios sobre AF e saúde cardiometabólica. ${ }^{6}$

Em recente pesquisa feita em participantes do Estudo Longitudinal de Saúde do Adulto (ELSA-Brasil), os resultados mostraram que existe associação entre AF e maiores níveis de HDL-C, tanto em homens quanto em mulheres, Além disso, observou-se que a prática de AF em intensidade vigorosa associava-se com mudanças mais positivas nos perfis lipídicos do que apenas a duração da prática da AF. No entanto, nesse estudo, não foram analisadas possíveis diferenças entre homens e mulheres no que diz respeito à quantidade (tempo e duração) de AF. ${ }^{7}$

O conhecimento da existência de efeito dose-resposta entre a AF e o aumento do HDL-C irá agregar a produção de novas informações e pesquisas sobre o tema, considerando que as políticas públicas de promoção da AF poderão usar essas informações como base para novas propostas de intervenção. Além disso, apesar de a associação entre AF e os níveis de HDL-C ser conhecida, estudos que demonstrem o efeito dose-resposta no que diz respeito a possíveis diferenças entre homens e mulheres são escassos na literatura consultada sobre o assunto. Pesquisas que reafirmam a associação entre
AF e HDL-C ainda são de suma importância para um melhor entendimento dessa associação, principalmente ao se analisar as possíveis diferenças entre sexos nesses achados.

O objetivo do estudo foi verificar a existência de diferenças entre homens e mulheres no efeito dose-resposta na associação entre AF e HDL-C entre participantes do ELSA-Brasil.

\section{Métodos}

\section{População e amostra}

O ELSA-Brasil é um estudo de coorte de 15.105 servidores públicos ativos ou aposentados na faixa etária de 35 a 74 anos de seis instituições de nível superior, localizadas nas cidades de Salvador, Vitória, Belo Horizonte, Rio de Janeiro, São Paulo e Porto Alegre, que tem como principal objetivo investigar a incidência e a progressão de diabetes e doenças cardiovasculares e seus fatores associados (biológicos, comportamentais, ambientais, ocupacionais, psicológicos e sociais), cujos detalhes metodológicos foram descritos previamente. ${ }^{8,9}$ Até o momento, já foram coletadas informações sobre os participantes em três oportunidades: 1ํㅡ onda, de 2008 até 2010; 2ª onda, de 2012 até 2014; e 3a onda, de 2016 até 2018. Para o presente estudo, foram selecionados todos os participantes da Onda 2 (2012-2014) que responderam aos questionários sobre AF e com dados completos relativos às variáveis envolvidas na análise, totalizando 13.931 participantes (7.607 mulheres).

O ELSA-Brasil foi aprovado pela Comissão Nacional de Ética em Pesquisa (CONEP) e em todos os Comitês de Ética em Pesquisa dos seis centros de investigações envolvidos. Todos os participantes assinaram o termo de consentimento livre e esclarecido, sendo garantidos o sigilo e a confidencialidade dos dados.

\section{Produção de dados}

Os dados foram coletados por uma equipe de entrevistadores e aferidores treinados e certificados por um comitê de controle de qualidade, ${ }^{9}$ capacitados a executar o protocolo do estudo em qualquer Centro de Investigação ELSA-Brasil. Realizaram-se entrevistas face a face na aplicação dos blocos de questionários para obtenção das informações sobre idade, escolaridade, renda familiar e tabagismo. Além disso, foram coletadas informações sobre medidas antropométricas de peso, estatura e circunferência da cintura. 
O peso corporal foi obtido pela manhã, após 8 a 12 horas de jejum e com o participante sem sapatos e com roupas leves. Foi usada balança eletrônica Toledo ${ }^{\circledR}$, com capacidade para até $200 \mathrm{~kg}$. Para medida da estatura, foi usado estadiômetro da marca $\mathrm{SECA}^{\circledR}$, com o participante posicionado de pé e seguindo rigorosamente o plano de Frankfurt. A obesidade foi identificada por meio do índice de massa corporal (IMC), aplicando-se a equação IMC = peso $(\mathrm{kg}) / \mathrm{altura}(\mathrm{m})^{2}$, adotandose o seguinte ponto de corte; obesidade $=0$ se IMC $<30,0$ e obesidade $=1$ se IMC $\geq 30,0$.

\section{Avaliação da atividade física}

Para identificação e quantificação da AF, foi utilizado o International Physical Activity Questionnary (IPAQ), versão longa, que é constituído de questões relativas à frequência e à duração de atividades físicas (caminhada, AF moderada e AF vigorosa) desenvolvidas no trabalho, no deslocamento, nas atividades domésticas e no tempo livre. ${ }^{10}$ No ELSA-Brasil, apenas os domínios do tempo livre e do deslocamento foram avaliados. A AF foi mensurada em minutos/semana por meio da multiplicação da frequência semanal pela duração de cada uma das atividades realizadas. Para efeito desse estudo, utilizou-se apenas a atividade física no tempo livre (AFTL), com a seguinte categorização: $0=$ inativo fisicamente $(<10$ minutos de qualquer AF na semana); 1 = pouco ativo (de 10 minutos a menos de 150 minutos de caminhada/moderada por semana e/ou de 10 minutos a menos que 60 minutos de atividades vigorosas por semana e/ou de 10 minutos a menos que 150 minutos por semana de qualquer combinação de caminhada, moderada e vigorosa); 2 = fisicamente ativo ( $\geq 150$ minutos de caminhada/atividade moderada por semana e/ou $\geq 60$ minutos de atividades vigorosas por semana e/ou $\geq 150$ minutos por semana de qualquer combinação de caminhada, moderada e vigorosa); 3 = muito ativo $(\geq 150$ minutos de atividades vigorosas por semana ou $\geq 60$ minutos de atividades vigorosas por semana +150 minutos por semana de qualquer combinação entre caminhada e moderada). Para as análises dicotomizadas, foram considerados como insuficientemente ativos aqueles classificados como inativos fisicamente e pouco ativos, e como ativos aqueles classificados como fisicamente ativos e muito ativos.

\section{Avaliação do HDL-C}

A coleta de sangue foi realizada após 12 horas de jejum durante a noite e centrifugadas e armazenadas em criotubos em temperatura de $-80^{\circ} \mathrm{C}$ nos próprios centros de investigação. Como forma de garantir a qualidade e a padronização dos resultados, todas as amostras foram enviadas para processamento e análise em um laboratório central do ELSA-Brasil localizado na cidade de São Paulo. Os valores de HDL-C em mg/dL foram determinados por um método colorimétrico homogêneo usando um equipamento ADVIA 1200 Siemens $^{\circledR}{ }^{7}$ O O HDL-C foi dicotomizado em $<60 \mathrm{mg} / \mathrm{dL}$ e $\geq 60 \mathrm{mg} / \mathrm{dL}$ (HDL-C elevado).

\section{Covariáveis}

Para as covariáveis, adotou-se a seguinte categorização: idade $=0$, se entre 34 e 50 anos; idade $=1$, se entre 51 e
60 anos; e idade $=2$, se $>60$ anos. Para o grau de instrução, foram estabelecidos quatro estratos: $0=$ fundamental incompleto; 1 = fundamental completo; 2 = médio completo; e 3 = superior completo/pós-graduação. A renda familiar foi categorizada em: até 2 salários mínimos $=0$; de 2 até 8 salários mínimos $=1$; de 8 até 18 salários mínimos $=2$; e acima de 18 salários mínimos $=3$. O tabagismo atual foi categorizado em não $=0$ e $\operatorname{sim}=1$.

\section{Análise dos dados}

As medidas descritivas (proporções) foram calculadas para todas as variáveis categorizadas. As análises foram estratificadas por sexo a priori e comparadas por meio do teste Qui-quadrado. Para testar o poder discriminatório das diferentes intensidades de AFTL (caminhada, AF moderada e AF vigorosa) para HDL-C elevado, foram utilizadas curvas ROC (Receiver Operating Characteristic). ${ }^{11}$ Utilizou-se intervalo de confiança (IC) a 95\%. As associações foram analisadas por meio de regressão logística. Foram consideradas como potenciais confundidoras ou modificadoras de efeito as seguintes variáveis: idade, obesidade, tabagismo no momento da entrevista, renda familiar e escolaridade. Foram selecionadas para modelagem as variáveis que, na etapa bivariada (matriz tetracórica), apresentaram na avaliação simultânea $p \leq 0,05$ e coeficiente de correlação rho $<0,60$.

A verificação da modificação do efeito foi feita por meio de estratificação com a observação das medidas pontuais estrato-específicas e os seus intervalos de confiança. Quando a medida pontual de um fator, em determinado estrato específico, não estava contida no intervalo de confiança do outro fator no mesmo estrato, isso indicava modificação de efeito. A análise das possíveis variáveis de confundimento foi realizada pelo procedimento backward, por meio de regressão logística. A análise começa com o modelo completo, seguido pela remoção um a um dos potenciais confundidores. Quando observada modificação igual ou superior a 10\% na associação pontual entre AFTL e HDL-C, a variável é considerada confundidora. ${ }^{12}$ No processo de modelagem, não foram identificadas variáveis modificadoras de efeito, e a variável idade foi identificada como confundidora. Portanto, o melhor modelo para análise da associação entre AFTL e HDL-C foi aquele ajustado por idade. Foi também analisado o efeito dose-resposta na associação entre AFTL e HDL-C. Para esta análise, foram criadas variáveis DUMMIES para comparação entre o grupo de referência (inativo fisicamente) e cada um dos outros estratos da variável AF (insuficientemente ativo, ativo e muito ativo). Foi utilizado o teste de Mantel-Haenszel para testar a homogeneidade dos valores da odds ratio (OR) entre os estratos de cada variável com nível de significância de 5\%. O intervalo de confiança foi estabelecido em 95\%. Empregou-se o programa estatístico STATA ${ }^{\circledR}$ versão 12.0 .

\section{Resultados}

Um total de 6.324 homens e 7.607 mulheres foi incluído na análise. As características da amostra estão apresentadas na Tabela 1. A média de idade entre as mulheres é de 55,7 $\pm 8,8$ e entre os homens de 55,7 $\pm 9,2$. Observa-se também que os homens têm maior renda familiar, são mais tabagistas e 


\begin{tabular}{|c|c|c|c|}
\hline & Homens $(n=6.324)$ & Mulheres $(n=7.607)$ & p \\
\hline \multicolumn{4}{|l|}{ Idade (anos) - n (\%) } \\
\hline $34-50$ & $2.055(32,5)$ & $2.342(30,8)$ & \\
\hline $51-60$ & $2.182(34,5)$ & $2.752(36,2)$ & \\
\hline$>60$ & $2.087(33,0)$ & $2.511(33,0)$ & 0,49 \\
\hline \multicolumn{4}{|l|}{$\begin{array}{l}\text { Renda familiar } \\
\text { (salário mínimo) - n (\%) }\end{array}$} \\
\hline Até 2 & $60(0,9)$ & $84(1,1)$ & \\
\hline De 2 até 8 & $2.117(33,6)$ & $2.545(33,6)$ & \\
\hline De 8 até 18 & $2.462(39,1)$ & $3.356(44,3)$ & \\
\hline Acima de 18 & $1.657(26,3)$ & $1.583(20,9)$ & $<0,01$ \\
\hline \multicolumn{4}{|l|}{ Escolaridade - n (\%) } \\
\hline Fundamental incompleto & $448(10,2)$ & $261(4,5)$ & \\
\hline Fundamental completo & $482(10,9)$ & $390(6,7)$ & \\
\hline Médio completo & $1.933(43,9)$ & $2.449(42,3)$ & \\
\hline Superior completo/Pós-graduação & $1.533(34,9)$ & $2.694(46)$, & $<0,01$ \\
\hline \multicolumn{4}{|l|}{ Obesidade - n (\%) } \\
\hline $\mathrm{IMC}<30 \mathrm{~kg} / \mathrm{m}^{2}$ & $4.763(75.3)$ & $5.308(69,8)$ & \\
\hline $\mathrm{IMC} \geq 30 \mathrm{~kg} / \mathrm{m}^{2}$ & $1.561(24,7)$ & $2.299(30,2)$ & $<0,01$ \\
\hline \multicolumn{4}{|l|}{ Tabagismo atual - $\mathrm{n}(\%)$} \\
\hline Não & $3.290(52,0)$ & $4.863(63,9)$ & \\
\hline $\operatorname{Sim}$ & $3.033(48,0)$ & $2.744(36,1)$ & $<0,01$ \\
\hline \multicolumn{4}{|l|}{ Atividade física no tempo livre - n (\%) } \\
\hline Inativo fisicamente & $2.218(35,1)$ & $3.401(44,7)$ & \\
\hline Insuficientemente ativo & $1.089(17,2)$ & $1.342(17,6)$ & \\
\hline Ativo & $1.749(27,6)$ & $1.947(25,6)$ & \\
\hline Muito ativo & $1.268(20,1)$ & $917(12,0)$ & $<0,01$ \\
\hline \multicolumn{4}{|l|}{ HDL-c elevado } \\
\hline Sim & $916(14,5)$ & $3.241(42,7)$ & \\
\hline Não & $5.408(85,5)$ & $4.366(57,3)$ & $<0,01$ \\
\hline
\end{tabular}

Valores para homens e mulheres foram comparados por meio do teste Qui-quadrado.

mais ativos no tempo livre, enquanto as mulheres são mais obesas e apresentam maior proporção de níveis de HDL-C mais elevados. Observa-se ainda que não existem diferenças estatisticamente significativas entre homens e mulheres com relação à idade.

Na Tabela 2, pode-se observar o poder discriminatório das diferentes intensidades de AFTL para o aumento do nível de HDL-C. Entre homens, apenas a AF vigorosa apresentou áreas sob a curva ROC estatisticamente significativas, enquanto, entre as mulheres, tanto a caminhada quanto as atividades físicas moderadas e vigorosas apresentaram significância estatística para discriminar o aumento do HDL-C; apesar das áreas sob as curvas ROC não apresentarem valores elevados.

A associação entre AFTL e HDL-C em indivíduos do sexo masculino e feminino está demonstrada na Tabela 3. Observase que homens ativos têm 38\% de chance de ter níveis mais altos de HDL-C, enquanto, nas mulheres ativas, a chance é de $41 \%$, em comparação aos homens e mulheres inativos fisicamente, respectivamente.

$\mathrm{Na}$ Tabela 4, quando a AF foi classificada em quatro categorias, podemos observar a existência de efeito doseresposta na associação entre AFTL e HDL-C, tanto em homens quanto em mulheres. Contudo, entre os indivíduos do sexo masculino, a associação foi encontrada apenas naqueles classificados como ativos e muito ativos fisicamente.

\section{Discussão}

O estudo analisou a existência de efeito dose-resposta na associação entre AFTL e o aumento do HDL-C.

É importante salientar que a população do estudo se constitui de uma coorte de voluntários de servidores públicos, que, apesar de não ser representativa da população de modo geral, apresenta um número significativo de participantes em seis capitais brasileiras, fato que representa ponto forte do estudo

Os mecanismos que relacionam a maior intensidade de AF e mudanças lipídicas mais positivas são explicados em função de que, durante a AF, há aumento da atividade da LPL e do catabolismo dos triglicérides que resultam na transferência de colesterol, fosfolipídios e proteínas para as partículas nascentes do HDL-C, aumentado assim sua concentração. ${ }^{4,5}$ Além disso, o tempo médio de vida das partículas de HDL-C é maior em indivíduos mais ativos fisicamente, fato que pode contribuir para que o transporte reverso do colesterol possa ser maximizado. ${ }^{5}$

Neste contexto, em pesquisa realizada com participantes do ELSA-Brasil, demonstrou-se que há uma associação benéfica entre os níveis de AF e perfil lipídico favorável para HDL-C e triglicérides, tanto em homens quanto em mulheres. Além disso, observou-se que quanto maior fosse a intensidade da $A F$, mais positivas seriam as mudanças no perfil lipídico. ${ }^{7}$ 
Tabela 2 - Áreas sob as curvas ROC para diferentes intensidades de atividade física como discriminador de HDL-c elevado ( $\geq 60 \mathrm{mg} / \mathrm{dL}$ )

\begin{tabular}{llc}
\hline Atividade física no tempo livre & Masculino & Feminino \\
\hline Caminhada & $0,52(0,50-0,54)$ & $0,52(0,51-0,53)^{*}$ \\
\hline Moderada & $0,52(0,50-0,54)$ & $0,53(0,53-0,54)^{*}$ \\
\hline Vigorosa & $0,54(0,52-0,56)^{*}$ & $0,53(0,52-0,54)^{*}$ \\
\hline
\end{tabular}

ROC: receiver operating characteristic; IC95\%: intervalo de confiança a 95\%. *Área sob a curva ROC apresentando poder discriminatório IM (Li-IC >0,50).

Tabela 3 - Associação entre atividade física no tempo livre e HDL-c

\begin{tabular}{lcc}
\hline Atividade física no tempo livre & Masculino & Feminino \\
\hline $\begin{array}{l}\text { Insuficientemente ativos } \\
\text { (Inativos fisicamente e pouco ativos) }\end{array}$ & 1,00 & 1,00 \\
Ativos & $1,38(1,20-1,59)$ & $1,41(1,29-1,55)$ \\
(Ativos e muito ativos fisicamente) & \\
\hline
\end{tabular}

${ }^{*}$ Ajustado por idade

Tabela 4 - Efeito dose-resposta na associação entre atividade física no tempo livre e HDL-c

\begin{tabular}{lcc}
\hline Atividade física no tempo livre & Masculino & Feminino \\
\hline Inativo & 1,00 & 1,00 \\
Pouco ativo & $1,21(0,98-1,50)$ & $1,15(1,01-1,31)$ \\
Ativo & $1,27(1,05-1,52)$ & $1,33(1,19-1,49)$ \\
Muito ativo & $1,77(1,46-2,15)$ & $1,81(1,56-2,09)$ \\
\hline
\end{tabular}

* Ajustado por idade.

Em outro estudo, realizado com indivíduos residentes de Caxias do Sul/RS com mais de 60 anos de idade, foi demonstrado que aqueles que praticavam atividades físicas de intensidade moderada tinham níveis de LDL-c reduzidos e HDL-C aumentados quando comparados com os idosos que tinham baixos níveis de $\mathrm{AF}^{13}$

Segundo Matsudo et al., ${ }^{14}$ existem poucas evidências a respeito dos mecanismos responsáveis pela redução dos níveis de LDL-c e lipoproteína de muito baixa densidade (VLDL-c). Contudo, a principal razão para elevação do HDL-C é a maior ação da LPL em resposta a exercícios físicos, fato que aumenta o catabolismo dos triglicérides e provoca a transferência de colesterol, fosfolipídios e proteínas para as partículas de HDL-C, aumentando assim a sua concentração. ${ }^{14}$ Outros estudos demonstraram que homens e mulheres ativos fisicamente têm maiores níveis de HDL-C e menores níveis de LDL-c e VLDL-c que seus pares inativos fisicamente, ${ }^{15}$ além de que, em outro trabalho realizado apenas com pessoas do sexo feminino, foi observado que há diferenças significativas nos níveis de HDL-C em mulheres com alta, moderada e baixa aptidão física, e os valores mais altos foram encontrados nas mulheres com níveis altos de aptidão física. ${ }^{16}$

É importante enfatizar também que, no nosso estudo, a intensidade de AFTL foi classificada em caminhada, AF moderada e AF vigorosa. Neste contexto, apesar de as áreas sob as curvas ROC não apresentarem valores elevados, entre os homens, apenas a AF vigorosa discrimina o HDL-C elevado, enquanto, entre as mulheres, tanto a caminhada quanto a AF moderada ou vigorosa discriminam o HDL-C elevado, demonstrando que os homens precisam de AF mais intensa para aumentar os níveis de HDL-C.

Mais um aspecto importante a ser destacado, no presente estudo, é que o efeito dose-resposta entre AFTL e HDL-C tem significância tanto nas mulheres classificadas como pouco ativas quanto nas mulheres classificadas como ativas e muito ativas. Nos homens, por sua vez, a associação é observada apenas naqueles classificados como ativos e muito ativos. Desta forma, é possível compreender que eles têm a necessidade de praticar maior quantidade de AF para que tenham um resultado positivo quanto ao aumento do HDL-C.

Em recente publicação, também com dados do ELSABrasil, com objetivo de verificar a associação entre AFTL e AF no deslocamento com escores de risco cardiovascular, foi observado que apenas a AFTL estava inversamente associada com os eventos cardiovasculares, além de que os homens necessitavam de maior quantidade de AF (duração e intensidade), também com efeito dose-resposta, do que as mulheres para que os benefícios fossem alcançados. ${ }^{17}$

Esses resultados podem ser parcialmente explicados pelo fato de que, nos homens, os parâmetros homeostáticos em repouso - tais como frequência cardíaca, pressão arterial, níveis glicêmicos, gasto calórico, entre outros - são mais elevados do que nas mulheres, apesar de que, na pósmenopausa e com o processo do envelhecimento, os valores de pressão arterial podem ser maiores no sexo feminino. ${ }^{18,19}$ Assim, para quebrar a homeostase de repouso, os homens precisam de maior quantidade de AF (duração e intensidade) do que as mulheres, para, com isso, fazer com que possam 
ser ativados os mecanismos que desencadeiam a proteção cardiovascular, tais como redução da pressão arterial, redução dos níveis glicêmicos e de triglicérides, além do aumento do HDL-C (que, no repouso, apresenta menores níveis nos homens. ${ }^{6}$

Uma possível limitação pode ser atribuída à informação sobre $\mathrm{AF}$, pois a mesma foi obtida por meio de questionários que, apesar de ser um instrumento largamente utilizado em estudos nacionais e internacionais, pode apresentar problemas como, por exemplo, o viés de memória. Ademais, é importante ressaltar que, no presente estudo, foi analisada apenas a AFTL, não sendo considerados os outros domínios da AF, como, por exemplo, deslocamento, AF no trabalho e AF doméstica. Além disso, considerando que se trata de estudo transversal, a causalidade reversa pode limitar a força dos resultados.

\section{Conclusão}

De acordo com os resultados observados no presente estudo, sugere-se que a prática da AF discrimina e está associada a níveis mais elevados de HDL-C em adultos de ambos os sexos. É importante ressaltar que, nos homens, existe a necessidade de maior quantidade de atividades físicas (duração e intensidade) para que os benefícios possam ser alcançados. Esses resultados são de grande importância para a saúde pública, considerando que as políticas públicas de promoção de atividades físicas podem ser norteadas com base nessas informações. Sugerem-se estudos longitudinais sobre o tema para que se possa confirmar os presentes resultados.

\section{Referências}

1. Haskell WL. The influence of exercise on the concentrations of triglyceride and cholesterol in human plasma. Exerc Sport Sci Rev. 1984; 12:205-44.

2. Rader DJ, Hovingh GK. HDL and cardiovascular disease. Lancet. 2014; 384(9943):618-25. doi:10.1016/S0140-6736(14)61217-4.

3. Voight BF, Peloso GM, Orho-Melander M, Frikke-Schmidt R, Barbalic M, Jensen MK,et al. Plasma HDL cholesterol and risk of myocardial infarction: a mendelian randomisation study [published correction appears in Lancet. 2012 Aug 11;380(9841):564]. Lancet. 2012; 380(9841):572-80. doi:10.1016/S0140-6736(12)60312-2.

4. Pitanga FJG. Atividade física e lipoproteínas em adultos de ambos os sexos, Rev Bras Ciênc Mov. 2001; 9(4):25-31. doi:http://dx.doi.org/10.18511/ rbcm.v9i4.402.

5. Hebert PN, Bernier DN, Cullianane EM,Edelstein L, Kantor MA, et al. Highdensity lipoprotein metabolism in runners and sedentary men. JAMA. 1984; 252(8):1034-7.

6. Pitanga FJG, Pitanga CPS, Beck CC. Physical activity for the prevention of cardiometabolic diseases: how much is required? Curr Res Diabetes Obes J. 2019; 9(4):17-32. doi:10.19080/CRDOJ.2019.09.555766.

7. Silva RC, Hausein MF, Diniz S, Alvim S, Vidigal PG, Fedeli LMG, et al. Atividade física e perfil lipídico no estudo longitudinal de saúde do adulto (ELSA-Brasil). Arq Bras Cardiol. 2016; 107(1):10-9. doi: 10.5935/ abc. 20160091

8. Aquino EM, Barreto SM, Bensenor IM, Carvalho MS, Duncan BB, Lotufo PP, et al. Brazilian Longitudinal Study of Adult Health (ELSA-Brasil): objectives and design. Am J Epidemiol. 2012; 175(4):315-24. doi: 10.1093/aje/ kwr294.

\section{Contribuição dos autores}

Concepção e desenho da pesquisa, Obtenção de dados e Obtenção de financiamento: Griep RH, Almeida MC, Fonseca MJM, Matos SMA; Análise e interpretação dos dados: Pitanga FJG, Griep RH, Almeida MC, Fonseca MJM, Souza AR, Silva RC, Matos SMA; Análise estatística: Pitanga FJG, Almeida MC; Redação do manuscrito: Pitanga FJG, Souza AR, Silva RC; Revisão crítica do manuscrito quanto ao conteúdo intelectual importante: Pitanga FJG, Griep RH, Almeida MC, Fonseca MJM, Matos SMA.

\section{Potencial conflito de interesse}

Não há conflito com o presente artigo

\section{Fontes de financiamento}

O presente estudo foi financiado pelo Ministério da Saúde do Brasil (Departamento de Ciência e Tecnologia) e o Ministério de Ciência e Tecnologia da Brasil (Financiadora de Estudos e Projetos e CNPq - Conselho Nacional de Pesquisa), processos no 01 060010.00RS, 0106 0212.00BA, 0106 0300.00ES, 0106 0278.00MG, 0106 0115.00SP, 01 $060071.00 \mathrm{RJ}$

\section{Vinculação acadêmica}

Não há vinculação deste estudo a programas de pósgraduação.

9. Schimidt MI, Griep RH, Passos VM, Luft C, Goulart AC, Menezes GM, et al. Strategies and development of quality assurance and control in the ELSABrasil. Rev Saude Publica. 2013; 47(Suppl 2):105-12. doi: 10.1590/S00348910.2013047003889.

10. Matsudo S, Araújo T, Matsudo V. Questionário internacional de atividade física (IPAQ): Estudo de validade e reprodutibilidade no Brasil. Rev Bras. Ativ. Fís. Saude. 2001; 6(2):5-18. doi:https://doi.org/10.12820/rbafs.v.6n2p5-18

11. Erdreich LS, Lee ET. Use of relative operating characteristics analysis in epidemiology: a method for dealing with subjective judgement. Am J Epidemiol .1981;114(5):649-62. doi:https://doi.org/10.1093/ oxfordjournals.aje.a113236

12. Hosmer JR, Lemeshow S. Appled logistic regression. 2 ed. New York: John Wiley \& Sons; 1989.

13. Ferreira APS, Picolli T, Bordin A. Baixos níveis de atividade física estão associados a prejuízos no perfil lipídico e aumento do percentual de gordura de indivíduos idosos. Rev Bras Ciênc Mov. 2015; 23(3):135-42. doi: http:// dx.doi.org/10.18511/0103-1716/rbcm.v23n3p135-142.

14. Matsudo VKR, Matsudo SMM, Araújo TL. Dislipidemias e a promoção da atividade física: uma revisão na perspectiva de mensagens de inclusão,.R Bras Cienc Mov. 2005;13(2):161-70. doi: http://dx.doi.org/10.18511/rbcm. v13i 2.638

15. Campaigne BN. Fontaine RN, ParK S, Rymaszewski ZJ. Reverse cholesterol transport with acute exercise. Medic Scienc Esport Exerc.1993; 25(12): 1346-51. 
16. Kokkinos PF, Holland JC, Pittaras AE, Narayan P, Dotson CO, Papademetriou $\mathrm{V}$, et al. Cardiorespiratory fitness and coronary heart desease risk factor association in women. J Am Coll Cardiol. 1995; 26(2): 358-64. doi: 10.1016/0735-1097(95)80007-4

17. Pitanga FJG, Matos SMA, Almeida MDC, et al. Leisure-Time Physical Activity, but not Commuting Physical Activity, is Associated with Cardiovascular Risk among ELSA-Brasil Participants. Arq Bras Cardiol. 2018;110(1):36-43. doi:10.5935/abc.20170178.
18. Choi HM, Kim HC, Kang DR. Sex differences in hypertension prevalence and control: Analysis of the 2010-2014 Korea National Health and Nutrition Examination Survey. PLoS One. 2017; 12(5):e0178334. doi:10.1371/ journal.pone.0178334.

19. Reckelhoff JF. Gender differences in the regulation of blood pressure. Hypertension. 2001; 37(5):1199-208. doi:10.1161/01.hyp.37.5.1199. 